\title{
A Relativistic Model for Isobar Configurations in the Two-Nucleon System
}

\author{
H. Arenhövel \\ Institut für Kernphysik der Universität Mainz, West Germany \\ (Z. Naturforsch. 32 a, 244-251 [1977] ; received January 20, 1977)
}

\begin{abstract}
A relativistic description of isobar configurations in the two-nucleon system is obtained by phenomenologically extending the Bethe-Salpeter-equation to channels comprising configurations of internally excited nucleons. The generalized equation is then reduced by standard methods to a three-dimensional relativistic equation. Various possible off-mass-shell extrapolations involved in this reduction are discussed. The best choice seems to be where the relative energy of the two particles is fixed to zero. Then all diagonal and transition OBE-interactions do not contain any retardation and have the normal Yukawa range.
\end{abstract}

\section{Introduction}

Virtual baryon resonance admixtures in nuclei, the so called nuclear isobar configurations (IC) have recently turned out to be quite interesting constituents of nuclei in the realm of intermediate energy physics. Quite a few theoretical and experimental studies have been conducted to shed light on those interesting questions as to the strengths of such isobar configurations and which nuclear properties or reactions with nuclei are influenced by them. Reviews may be found in Refs. ${ }^{1-3}$.

The most spectacular reaction in this respect has been so far the so called spectator isobar production at the deuteron to determine the content of the deuteron's double $\Delta(1236)$ component ${ }^{3,4}$. In this reaction a particle of sufficiently high energy kicks out one of the 4 's in the forward direction while the other "spectator" $\Delta$ recoils in the backward hemisphere. However, also in other reactions like elastic electron scattering from the deuteron and electroand photodisintegration of the deuteron the isobar configurations have been demonstrated to give quite substantial contributions ${ }^{5}$. Interesting effects in nuclear matter resulting from intermediate virtual $\mathrm{N} \Delta$ and $\Delta \Delta$ configurations are discussed in Ref. ${ }^{6}$.

Despite these different studies the exact strength of isobar configurations, in particular of the double$\Delta$-component in the deuteron, is still an open question. Experimental and theoretical estimates vary between less than 0.3 to 3 percent ${ }^{4,7,8}$. It has been shown that the main uncertainties in the theoretical predictions do not come from the computational methods used but rather from unknown properties of the transition and diagonal potentials of the isobar configurations and from rather crude model assumptions, essentially non-relativistic treatment and off-mass-shell problems ${ }^{9}$. The latter are expected to be much more important for IC than for nucleons in their internal ground state, because the virtual $\mathrm{N}^{*}$ 's are by several hundreds $\mathrm{MeV}$ off-massshell. However, without any detailed dynamical description of the nucleon internal degrees of freedom it is difficult to investigate the off-mass-shell effects.

Also the assumption of nonrelativistic kinematics is much less justified for IC than for conventional nuclear physics in view of the on the average higher momentum components of IC corresponding to velocities of about $v / c \approx 0.3-0.5$. Only in one case a relativistic description has been given by Weber ${ }^{8}$ using a relativistic three-body equation for the $(\mathrm{NN} \pi)$-channel with a quasi-particle approximation for the $(\mathrm{N} \pi)$-system. The results obtained were qualitatively not so different from the nonrelativistic treatment.

The aim of the present paper is also to arrive at a relativistic description of isobar configurations in the two-nucleon system. However, we shall use a somewhat different approach. Instead of considering an intermediate three-particle $(\mathrm{NN} \tau)$-system we shall phenomenologically extend the relativistic Bethe-Salpeter-equation to include internal nucleon degrees of freedom by admitting additional channels to the Bethe-Salpeter-amplitude corresponding to intrinsically excited nucleons, i.e., isobar configurations. This will be outlined in Section 2. In Sect. 3 we will discuss several approximations of the BetheSalpeter-equation for isobar configurations in order to obtain a relativistic three-dimensional description. A crucial point in these approximations is the question of how to fix the relative energy $p_{10}-p_{20}$ of the two particles corresponding to the relative 
time $t_{1}-t_{2}$. It is closely related to the off-mass-shell problems. In the final section different off-mass-shell extrapolations will be discussed in detail.

\section{Relativistic Bethe-Salpeter-equation for Isobar Configurations}

A wide class of three-dimensional relativistic models for a two-particle system starts from the fourdimensional relativistic Bethe-Salpeter-equation ${ }^{10}$ for the two-body scattering amplitude $M\left(q^{\prime}, q \mid P\right)$

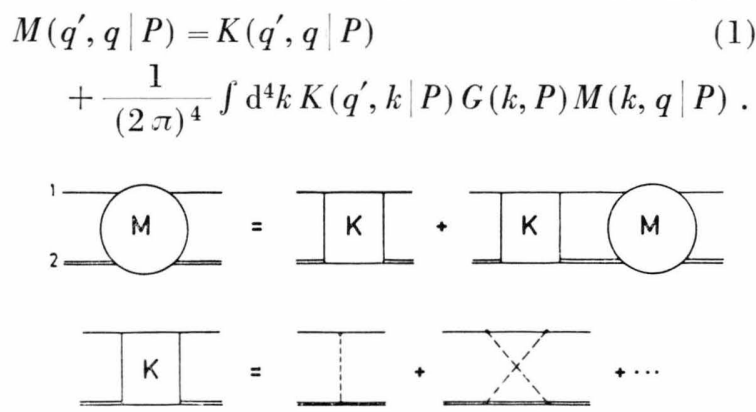

Fig. 1. Graphical representation of the Bethe-Salpeter equation for two distinct particles. The dashed lines should comprise all possible exchanged bosons.

A graphical representation is given in Figure 1. Assuming that the particles interact by exchange of a boson, the interaction kernel $K$ contains all irreducible two-particle diagrams. In this case the particles are assumed not to change their internal structure during a collision, i.e., internal degrees of freedom are neglected except spin and isospin. Here, $q$ and $q^{\prime}$ denote the initial and final relative four momenta, and $P$ the total four momentum of the two particles.

$$
\begin{gathered}
q=\eta_{2} p_{1}-\eta_{1} p_{2}, \quad P=p_{1}+p_{2}, \\
\eta_{1}+\eta_{2}=1 .
\end{gathered}
$$

Using the stability conditions for the two-particles with masses $M_{1}, M_{2}$ and for the bound state $\left[s=P^{2}<\left(M_{1}+M_{2}\right)^{2}\right]$ one chooses

$$
\eta_{i}=M_{i} /\left(M_{1}+M_{2}\right), \quad i=1,2
$$

to avoid a displaced pole in the Wick rotation ${ }^{10}$. This choice has also the pleasant feature that it corresponds to the usual definition of the relative momentum of two nonrelativistic particles of unequal masses. Another choice, which has the same feature in the nonrelativistic limit, is to choose the relativistic analog of center-of-mass and relative variables ${ }^{11}$

$$
\begin{aligned}
q & =\left[\left(p_{2} \cdot P\right) p_{1}-\left(p_{1} \cdot P\right) p_{2}\right] / s \\
& =\left(p_{1}-p_{2}\right) / 2-\left(p_{1}{ }^{2}-p_{2}{ }^{2}\right) P / 2 s
\end{aligned}
$$

from which $q \cdot P=0$ follows. However, one has to be careful since in the $\mathrm{cm}$-system one always has $q_{0}=0$ leaving $p_{10}-p_{20}$ indeterminate. Usually one chooses equal time, i.e., $p_{10}=p_{20}$.

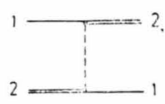

Fig. 2. Exchange interaction for two distinct particles.

Up to now we have assumed that the two interacting particles do not change their mass during the exchange of a boson mediating the interaction. However, if there is also an exchange interaction between the two particles (see Fig. 2), than one has to be careful in the case where the lowest mass $m_{\mathrm{B}}$ of the exchanged boson is smaller than the mass difference of the two particles $M_{2}-M_{1}$ assuming $M_{2}>M_{1}$. Because then the particle of mass $M_{2}$ is not stable if it is on the mass shell and for $s \geqq\left(2 M_{1}+m_{\mathrm{B}}\right)^{2}$ one would have to consider also coupling to the threeparticle state with one additional boson on-massshell. In this case the stability condition for the bound state is therefore $s<\left(2 M_{1}+m_{\mathrm{B}}\right)^{2}$ and one would choose

$\eta_{1}=M /\left(2 M_{1}+m_{\mathrm{B}}\right), \quad \eta_{2}=\left(M_{1}+m_{\mathrm{B}}\right) /\left(2 M_{1}+m_{\mathrm{B}}\right)$

to avoid the displaced pole. If $s<4 M_{1}{ }^{2}$ one could also choose $\eta_{i}=1 / 2$. This remark is of some importance if we now consider the two-nucleon system with special emphasis on the internal nucleon degrees of freedom. In the usual treatment the nucleons are assumed not to change their internal structure and all effects coming from excitation of internal degrees of freedom are taken into account by effective operators ${ }^{6,12}$. For instance, the interaction kernel then contains diagrams with intermediate isobars, i.e., nucleon resonances (Figure 3). Here

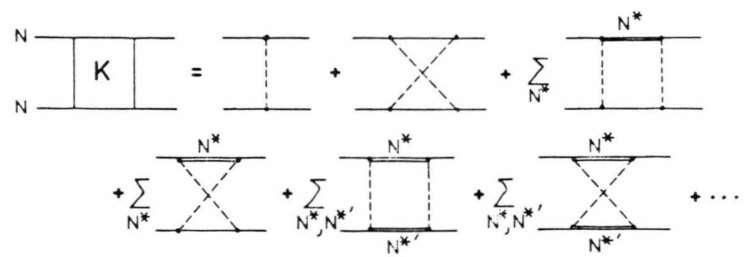

Fig. 3. Graphical representation of the effective interaction kernel of the two-nucleon system including intermediate isobar excitations. 
we shall pursue the alternative way of taking into account a possible excitation of internal nucleon degrees of freedom by including configurations of one or two excited nucleon states into the amplitudes and wave functions. This can be achieved by simply extending the matrices $M$ and $K$ which up to now have included only spin and isospin degrees of freedom to all possible combinations of internally excited states of the two particles. If one labels these different intrinsic configurations of the two particles by $n=\left\{n_{1}, n_{2}\right\}$ one obtains a simple generalization of the Bethe-Salpeter-equation

$$
\begin{aligned}
M_{n^{\prime} n}\left(q^{\prime}, q \mid P\right)= & K_{n^{\prime} n}\left(q^{\prime}, q \mid P\right) \\
& +\frac{1}{(2 \pi)^{4}} \sum_{n^{\prime \prime}} \int \mathrm{d}^{4} k K_{n^{\prime} n^{\prime \prime}}\left(q^{\prime}, k \mid P\right) \\
& \cdot G_{n^{\prime \prime}}(k, P) M_{n^{\prime \prime} n}(k, q \mid P)
\end{aligned}
$$

where $M_{n^{\prime} n}$ describes the off-shell scattering amplitude of an incoming two-particle configuration $n$ leading to an outgoing two-particle configuration $n^{\prime}$. For on-mass-shell particles one would in general violate unitarity since coupling to other open manyparticle channels are neglected. However, at present we are interested in two-nucleon states below pion production threshold, where no three particles states are open. That means, we only have to insure two particle unitarity.

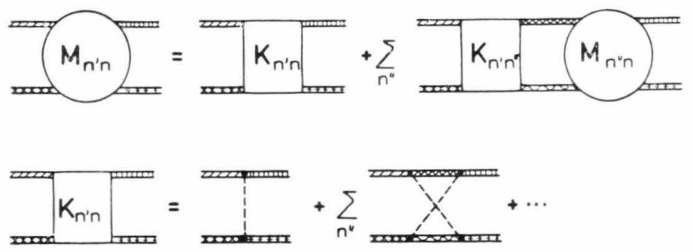

Fig. 4. Graphical representation of the generalized BetheSalpeter equation for isobar configurations. The different shadings should indicate different isobars.

A graphical representation is given in Figure 4. In particular, we note that some irreducible diagrams of Fig. 3 are now reducible. With respect to the above remark we will choose $\eta_{i}=1 / 2$ for all configurations $n$ because the mass of the particles can change during a collision and the energetically lowest configuration is the symmetric two-nucleon configuration. The physical picture behind this choice is that irrespective of the momentary intrinsic mass of one particle the various exchange interactions will distribute the total energy of the system evenly between the two particles. Then the lowest order diagram of the interaction kernel constituting the OBE-part is given by

$$
V_{n^{\prime} n}^{(2)}\left(q^{\prime}, q \mid P\right)=\sum_{B} \frac{\Gamma_{n^{\prime} n}\left(q^{\prime}, q ; P\right)}{\left(q^{\prime}-q\right)^{2}-m_{\mathrm{B}}{ }^{2}}
$$

where $\Gamma_{n^{\prime} n}^{\mathrm{B}}$ denotes the appropriate vertex functions, which are obtained from some effective model interaction-Lagrangian. In general they contain also unknown form factors for the case that the particles involved in the vertex are off-mass-shell. This poses a serious problem for any off-mass-shell extrapolation.

\section{Approximation by a Three-dimensional Relativistic Equation}

To obtain from this four-dimensional equation a relativistic three-dimensional equation one usually replaces the two-particle Green's function $G$ by another two-particle Green's function $g$ which produces only two-particle cuts in the physical region, i.e., when both particles are on-shell ${ }^{13}$. Furthermore, the relative energy $q_{0}$ is fixed by some prescription depending on the off-mass-shell extrapolation chosen. One obtains in the first step an equation similar to the Bethe-Salpeter-equation

$$
\begin{aligned}
M_{n^{\prime} n}\left(q^{\prime}, q \mid P\right)= & W_{n^{\prime} n}\left(q^{\prime}, q \mid P\right) \\
& +\frac{1}{(2 \pi)^{4}} \sum_{n^{\prime \prime}} \int \mathrm{d}^{4} k W_{n^{\prime} n^{\prime \prime}}\left(q^{\prime}, k \mid P\right) \\
& \cdot g_{n^{\prime \prime}}(k, P) M_{n^{\prime \prime} n}(k, q \mid P)
\end{aligned}
$$

where the effective interaction kernel $W$ is obtained from a complicated integral equation

$$
W_{n^{\prime} n}=K_{n^{\prime} n}+\sum_{n^{\prime \prime}} K_{n^{\prime} n^{\prime \prime}}\left(G_{n^{\prime \prime}}-g_{n^{\prime \prime}}\right) W_{n^{\prime \prime} n} .
$$

This equation formally is still equivalent to the original Bethe-Salpeter-equation (7). To obtain an approximate three-dimensional equation the relative energies $q_{0}{ }^{\prime}, q_{0}$ and $k_{0}$ are fixed by some specifically chosen off-mass-shell extrapolation. To solve this equation is certainly as complicated as solving directly the original equation (7). However, Eq. (10) is usually expanded in powers of coupling constants and one restricts oneself to the lowest order terms. One has up to fourth order

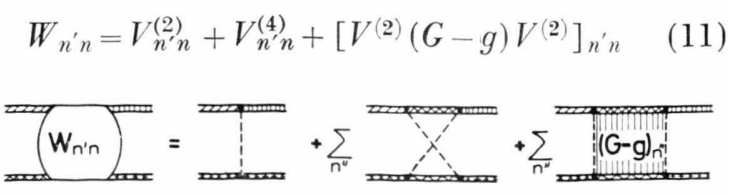

Fig. 5. The lowest order diagrams of the effective interaction kernel W for isobar configurations. 
(see Figure 5). The second part of the fourth-order term corresponding to an iteration of $V^{(2)}$ with an intermediate propagator $G-g$ involves off-shell properties of $V^{(2)}$. The hope is that with a proper choice of $g$ one can keep this term small. The restriction to $V^{(2)}$ constitutes the one-boson exchange model.

It is well known that this procedure of threedimensional reduction of the Bethe-Salpeter-equation is not unique, not only because of different prescriptions to fix the relative energy but also because of different possible choices of $g$. In fact, one has an infinite number of choices ${ }^{13}$. We shall first discuss two possible choices for fixing $q_{0}$ which are in use for the two-nucleon system:

(i) One particle is put on the mass shell, i.e., $p_{10}=E_{1 q}$ and $q_{0}=E_{1 q}-1 / 2 \sqrt{S}$. Such a prescription has been considered, for example, by Gross ${ }^{14}$ and by Erkelenz and Holinde ${ }^{15}$ for the two-nucleon system. An unpleasant feature of this approach is the unsymmetrical treatment of the two particles which has to be taken care of in antisymmetrizing the wave function. It seems natural that this choice is the appropriate one for isobar configurations in connection with spectator isobar production, where the deuteron is split up by a particle of high energy knocking out one constituent in the forward direction and leaving back a spectator isobar on-massshell recoiling in the backward direction. However, this approach which is quite useful in the pure twonucleon case will lead to serious problems if extended to isobar configurations as will be discussed soon.

(ii) The other widely used approach has first been discussed by Blankenbecler and Sugar ${ }^{16}$. In this case the relative energy is fixed by $q_{0}=0$ thus giving both particles the same energy. One could also interpret this as putting both particles equally off-mass-shell. Then one has

where

$$
p_{10}-E_{1 \underline{p}}=p_{20}-E_{2 \underline{p}}
$$

$$
E_{i \underline{p}}=\sqrt{\underline{\sim}^{2}+M_{i}^{2}} .
$$

This gives for the relative energy

$$
q_{0}=\left(E_{1 q}-E_{2 q}\right) / 2 .
$$

If the particles have the same mass one again has $q_{0}=0$. However, for configurations with unequal masses the relative energy does not vanish. It seems reasonable to consider the approach where both particles have the same energy $\left(q_{0}=0\right)$ irrespective of the intrinsic state for processes where the isobar configurations remain virtual, i.e., are not brought on the mass shell.

Before we look into the consequences of the different approaches, we will outline a widely used reduction to a relativistic three-dimensional equation. We shall start from following form for the two-particle Greens-function $g_{n}$ which will allow us to treat the different cases at once

$$
\begin{gathered}
g_{n}\left(k, P ; \alpha_{n}, \beta_{n}\right)=-2 \pi \int_{\left(M_{n_{1}}+M_{\left.n_{2}\right)^{2}}\right.} \frac{\mathrm{d} s^{\prime}}{s^{\prime}-s \varepsilon} \\
\cdot g_{n_{1}}^{(+)}\left(\alpha_{n} P^{\prime}+\beta_{n} P+k\right) g_{n_{2}}^{(+)}\left[\left(1-\alpha_{n}\right) P^{\prime}-\beta_{n} P-k\right]
\end{gathered}
$$

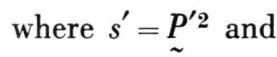

$$
g_{n_{i}}^{(+)}(p)=\frac{M_{n_{i}}}{E_{n_{i} \underline{p}}}\left(E_{n_{i} \underline{\sim}}+p_{0}\right) \Lambda_{n_{i}}^{+}(\underset{\sim}{p}) \delta^{(+)}\left(p^{2}-M_{n_{i}}^{2}\right) \text {. }
$$

Here, $M_{n}$ is the mass of the $i$-th particle in the configuration $n$,

$$
E_{n_{i} \underline{p}}=\sqrt{\underline{\sim}^{2}+M_{n_{i}}^{2}}
$$

and $\Lambda^{(+)}(p)$ projects onto positive energy oneparticles states of three-momentum $p$, i.e., only positive energy states are considered. The parameters $\alpha_{n}$ and $\beta_{n}$ may depend on $p_{1}$ and $p_{2}$ but on-mass-shell normalization would require $\alpha_{n}+\beta_{n}=1 / 2$ if both particles are on-mass-shell, i.e., $p_{1}{ }^{2}=M_{1}{ }^{2}$ and $p_{2}{ }^{2}=M_{2}{ }^{2}$. However, this applies only to the NNchannel since we will restrict ourselves to the region below pion production threshold, i.e., $s<2 M_{\mathrm{N}}+m_{\pi}$. Different choices of the parameters $\alpha_{n}$ and $\beta_{n}$ correspond to different off-mass-shell extrapolations, e.g., $\alpha_{n}=1 / 2, \beta_{n}=0$ gives in the two-nucleon case the Blankenbecler-Sugar equation while $\alpha_{n}=0$, $\beta_{n}=1 / 2$ puts particle " 1 " on-mass-shell. Before discussing the different possibilities mentioned above we will first proceed in the formal development, in particular derive an ordinary Lippman-Schwinger equation. Going into the $\mathrm{cm}$-frame and using

$$
\begin{aligned}
\delta^{(+)}\left[\left(\alpha_{n} P^{\prime}+\beta_{n} P+k\right)^{2}-M_{n_{1}}^{2}\right] \delta^{(+)}\left(\left[\left(1-\alpha_{n}\right) P^{\prime}-\beta_{n} P-k\right]^{2}-M_{n_{2}}^{2}\right) \\
=\frac{1}{8 E_{n_{1} k} E_{n_{2} k}} \delta\left(\frac{1}{2} P_{0}^{\prime}-E_{n \underline{\sim}}^{+}\right) \delta\left[k_{0}+\beta_{n} P_{0}-E_{n \underline{\sim}}^{-}\left(\alpha_{n}\right)\right]
\end{aligned}
$$


one obtains

$$
g_{n}\left(k, P ; \alpha_{n}, \beta_{n}\right)=-2 \pi \frac{M_{n_{1}} M_{n_{2}} E_{n k}^{+}}{E_{n_{1} k} E_{n_{2} \underline{\sim}}} \frac{\Lambda_{n_{1}}^{+}(\underset{\sim}{k}) \Lambda_{n_{2}}^{+}(\underset{\sim}{-k})}{\left(E_{n \sim}^{+}\right)^{2}-\frac{1}{4} P_{0}^{2}-i \varepsilon} \delta\left[k_{0}-E_{n \underline{\sim}}^{-}\left(\alpha_{n}\right)+\beta_{n} P_{0}\right]
$$

where we have set

$$
E_{n \underset{\sim}{k}}^{+}=\left(E_{n_{1} \underset{\sim}{k}}+E_{n_{2} k}\right) / 2, \quad E_{n \sim \sim}^{-}\left(\alpha_{n}\right)=\left(1-\alpha_{n}\right) E_{n_{1} \underset{\sim}{k}}-\alpha_{n} E_{n_{2} \underline{\sim}} .
$$

This means only positive energy states will occur. Inserted into Eq. (9) this finally gives for the scattering amplitude in the cm-frame

$$
\begin{aligned}
& M_{n^{\prime} n}\left(q_{0}{ }^{\prime}, q^{\prime}, q_{0}, \underset{\sim}{q} \mid P_{0}\right)=W_{n^{\prime} n}\left(q_{0}^{\prime}, q^{\prime}, q_{0}, \underset{\sim}{q} \mid P_{0}\right)-\frac{1}{(2 \pi)^{3}} \sum_{n^{\prime \prime}} \int \mathrm{d}^{3} k W_{n^{\prime} n^{\prime \prime}}\left(q_{0}{ }^{\prime}, \underset{\sim}{q^{\prime}}, k_{0}, \underset{\sim}{k} \mid P_{0}\right)
\end{aligned}
$$

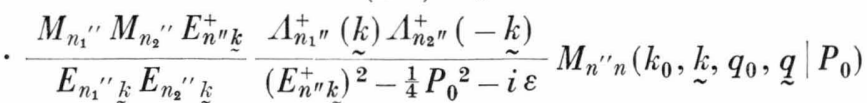

where the relative energies $q_{0}, q_{0}{ }^{\prime}$ and $k_{0}$ are fixed by

$$
q_{0}=E_{n q}^{-}\left(\alpha_{n}\right)-\beta_{n} P_{0}
$$

and correspondingly for $q_{0}{ }^{\prime}$ and $k_{0}$. To transform this equation into an ordinary Lippman-Schwinger equation one introduces as an effective scattering amplitude

$$
T_{n^{\prime} \lambda_{1}^{\prime} \lambda_{2}^{\prime}, n \lambda_{1} \lambda_{2}}\left(\underset{\sim}{q}, \underset{\sim}{q} \mid P_{0}\right)=N_{n^{\prime}}\left(q^{\prime}\right)\left\langle\lambda_{1}^{\prime} \lambda_{2}^{\prime}\left|M_{n^{\prime} n}\left(q_{0}^{\prime}, \underset{\sim}{q^{\prime}}, q_{0}, \underset{\sim}{q} \mid P_{0}\right)\right| \lambda_{1} \lambda_{2}\right\rangle N_{n}(\underset{\sim}{q})
$$

and an effective potential

$$
V_{n^{\prime} \lambda_{1}^{\prime} \lambda_{2}^{\prime}, n \lambda_{1} \lambda_{2}}\left(\underset{\sim}{q^{\prime}}, \underset{\sim}{q} \mid P_{0}\right)=N_{n^{\prime}}\left(\underset{\sim}{q^{\prime}}\right)\left\langle\lambda_{1}^{\prime} \lambda_{2}^{\prime}\left|W_{n^{\prime} n}\left(q_{0}^{\prime}, q^{\prime}, q_{0}, \underset{\sim}{q} \mid P_{0}\right)\right| \lambda_{1} \lambda_{2}\right\rangle N_{n}(\underset{\sim}{q})
$$

where the relative energies $q_{0}$ and $q_{0}{ }^{\prime}$ to be used in these expressions are given in Equation (22). Relativistic phase space corrections are contained in the factor

$$
N_{n}(q)=\left[2 E_{n \underline{q}}^{+} M_{n_{1}} M_{n_{2}} / E_{n_{1} \underline{q}} E_{n_{2} q}\left(M_{n_{1}}+M_{n_{2}}\right)\right]^{1 / 2} .
$$

Here $\lambda_{1}$ and $\lambda_{2}$ denote the helicities of particles one and two, respectively. The effective scattering amplitude $T$ obeys the ordinary LS-equation with the effective potential $V$.

$$
T_{n^{\prime} n}\left(q^{\prime}, q \mid P_{0}\right)=V_{n^{\prime} n}\left(\underset{\sim}{q^{\prime}}, q \mid P_{0}\right)-\frac{1}{(2 \pi)^{3}} \sum_{n^{\prime \prime}} \int \mathrm{d}^{3} k V_{n^{\prime} n^{\prime \prime}}\left(q^{\prime}, k \mid P_{0}\right) \frac{\frac{1}{2}\left(M_{n_{1}^{\prime \prime}}+M_{n_{2}^{\prime \prime}}\right)}{\left(E_{n^{\prime \prime} k}^{+}\right)^{2}-\frac{1}{4} P_{0}^{2}-i \varepsilon} T_{n^{\prime \prime} n}\left(\underset{\sim}{k} \underset{\sim}{q} \mid P_{0}\right) .
$$

Correspondingly one obtains from the equation for the bound state Bethe-Salpeter amplitude $\psi_{n}(q \mid P)$

$$
\psi_{n}(q \mid P)=\frac{1}{(2 \pi)^{4}} \sum_{n^{\prime}} \int \mathrm{d}^{4} k K_{n n^{\prime}}(q, k \mid P) G_{n^{\prime}}(k, P) \psi_{n^{\prime}}(k \mid P)
$$

the three-dimensional reduction

$$
\begin{aligned}
& \psi_{n}\left(q_{0}, \underset{\sim}{q} \mid P_{0}\right)=-\frac{1}{(2 \pi)^{3}} \sum_{n^{\prime}} \int \mathrm{d}^{3} k W_{n n^{\prime}}\left(q_{0}, \underset{\sim}{q}, k_{0}, \underset{\sim}{k} \mid P_{0}\right) \\
& \text { - } \frac{M_{n_{1}^{\prime}} M_{n_{2}^{\prime}} E_{n^{\prime} k}^{+k}}{E_{n_{1}^{\prime}{ }_{\sim}^{k}} E_{n_{2}^{\prime} \underset{\sim}{k}}} \frac{\Lambda_{n_{1}^{\prime}}^{+}(\underset{\sim}{k}) \Lambda_{n_{2}^{\prime}}^{+}(-\underset{\sim}{k})}{\left(E_{n^{\prime} k}^{+}\right)^{2}-\frac{1}{4} P_{0}^{2}-i \varepsilon} \psi_{n^{\prime}}\left(k_{0}, \underset{\sim}{k} \mid P_{0}\right)
\end{aligned}
$$

where $q_{0}$ and $k_{0}$ are given by Equation $(22)$. The transformation to an ordinary Schrödinger-type bound state equation is achieved by setting

$$
\varphi_{n \lambda_{1} \lambda_{2}}(\underset{q}{q})=\frac{\frac{1}{2}\left(M_{n_{1}}+M_{n_{2}}\right)}{\left(E_{n q}^{+}\right)^{2}-\frac{1}{4} P_{0}^{2}} N_{n}(q)\left\langle\lambda_{1} \lambda_{2}\left|\psi_{n}\left(q_{0}, \underset{\sim}{q}\right)\right| b\right\rangle
$$

and using again the effective potential $V$ of Equation (24). The bound state is denoted by $b$ and will be the deuteron state for the two-nucleon system. The helicity amplitudes $\varphi_{n \lambda_{1} \lambda_{2}}$ play the role of momentum space 
components of the deuteron wave funcion obeying an ordinary relativistic Schrödinger equation in momentum space

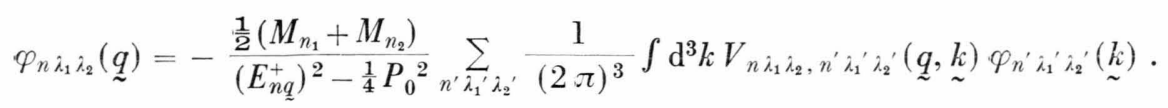

This constitutes a set of coupled equations for the various intrinsic components of the deuteron, i.e., normal part and isobar configurations.

To evaluate the denominator of Eq. (30) which contains the relativistic kinematics one has to distinguish between configurations where both particles have equal mass and those where the masses are different. In the former case one has

Then using

$$
\left(E_{n q}^{+}\right)^{2}=q^{2}+M_{n}^{2}, \quad M_{n_{1}}=M_{n_{2}}=M_{n} .
$$

$$
P_{0}^{2}=\left(2 M_{0}-B\right)^{2} \approx 4 M_{0}\left(M_{0}-B\right)
$$

where $M_{0}$ is the nucleon mass and $B$ the deuteron binding energy, one gets

$$
\left(E_{n q}^{+}\right)^{2}-\frac{1}{4} P_{0}^{2}=q^{2}+M_{n}^{2}-M_{0}^{2}+M_{0} B={\underset{\sim}{q}}^{2}+\left(M_{n}+M_{0}\right)\left(M_{n}-M_{0}\right)+M_{0} B .
$$

This is quite close to the expression in the nonrelativistic treatment where one uses $q^{2}+M_{n}\left[2\left(M_{n}-M_{0}\right)+B\right]$. In the other case, when the masses are different one obtains

$$
\left(E_{n q}^{+}\right)^{2}-\frac{1}{4} P_{0}^{2}=\frac{1}{2}{\underset{\sim}{q}}^{2}+\frac{1}{2}\left[\left(q^{2}+M_{n_{1}}^{2}\right)\left(q_{\sim}^{2}+M_{n_{2}}^{2}\right)\right]^{1 / 2}+\frac{1}{4}\left(M_{n_{1}}^{2}+M_{n_{2}}^{2}\right)-M_{0}^{2}+M_{0} B
$$

which in the nonrelativistic limit reduces to

$$
\begin{aligned}
\left(E_{n q}^{+}\right)^{2}-\frac{1}{4} P_{0}^{2} & =\underset{q^{2}}{q^{2}}+\frac{1}{4}\left[\left(M_{n_{1}}+M_{n_{2}}\right)^{2}-4 M_{0}{ }^{2}\right]+M_{0} B \\
& ={\underset{\sim}{q}}^{2}+\frac{1}{4}\left(M_{n_{1}}+M_{n_{2}}+2 M_{0}\right)\left(M_{n_{1}}+M_{n_{2}}-2 M_{0}\right)+M_{0} B, \quad q^{2} \ll M_{0} .
\end{aligned}
$$

The last expression is again very similar to the nonrelativistic expression $q^{2}+2 \mu_{n}\left(M_{n_{1}}+M_{n_{2}}-2 M_{0}+B\right)$ where $\mu_{n}$ denotes the reduced mass of configuration $n$.

\section{Discussion of Different Off-mass-shell Extrapolations}

The effective interaction kernel $W$ and thus the effective potential $V$ depend on the off-mass-shell extrapolation. We will discuss three cases:

(i) One particle is put on-mass-shell. Then the relative energy in a configuration $n$ is given by $\left(\alpha_{n}=0, \beta_{n}=1 / 2\right)$

$$
q_{0}=E_{n_{1} q}-\frac{1}{2} P_{0}
$$

and the energy transfer for a boson exchange between configurations $n$ and $n^{\prime}$ is

$$
q_{0}^{\prime}-q_{0}=E_{n_{1}^{\prime} q^{\prime}}-E_{n_{1} q} .
$$

One finds in some cases that the boson can be onmass-shell and that the corresponding potential is complex. For example, if particle " 1 " which is onmass-shell changes its intrinsic mass one has $\left(q^{\prime}-q\right)^{2}=m_{\mathrm{B}}{ }^{2}$ for $q^{\prime}=q$ with

$q_{\sim}^{2}=\left[\left(m_{\mathrm{B}}^{2}+M_{n_{1^{\prime}}}^{2}+M_{n_{2^{\prime}}}^{2}\right)^{2}-4 M_{n_{1^{\prime}}}^{2} M_{n_{2^{\prime}}}^{2}\right] / 4 m_{\mathrm{B}}^{2}$

if $M_{n_{1}}-M_{n_{1}}{ }^{\prime} \geqq m_{\mathrm{B}}$. In this case the transition potential becomes complex if the particle on-mass-shell changes its mass and if the mass difference is larger than the exchanged boson mass, an observation which has been made already by Partovi and Lomon ${ }^{17}$ who used a different $q_{0}$ as will be mentioned later. However, such a complex potential is rather artificial and unphysical because there is no decay channel with real bosons open in the case we consider here where the $N^{*}$ 's are virtual and therefore the potentials should be real. The origin of this pathology is the asymmetrical treatment of the two particles giving them rather different energies which in turn allows a large energy transfer by a boson exchange. Thus one should abandon this approach though it looked so appealing for the description of spectator isobar production. A possibility to keep this approach would be to take only the real part of the potentials in an ad hoc manner as proposed in Ref. ${ }^{17}$. However, this is not a satisfying procedure.

(ii) Both particles are equally put off-mass-shell. According to Eq. (13) this fixes the relative energy to

$$
q_{0}=\frac{1}{2}\left(E_{n_{1} q}-E_{n_{2} q}\right)
$$


which gives $\alpha_{n}=1 / 2$ and $\beta_{n}=0$. For equal mass configuration the relative energy vanishes but not for unequal mass configurations. Consequently, an exchanged boson transfers again not only momentum in this case but also energy if the initial or final configuration have unequal masses. The energy transfer is

$$
q_{0}^{\prime}-q_{0}=\frac{1}{2}\left(E_{n_{1}^{\prime} q^{\prime}}-E_{n_{2}^{\prime} q^{\prime}}-E_{n_{1} q}+E_{n_{2} q}\right) .
$$

This means that in some cases depending on the mass differences of the participating configurations again the exchanged boson can be on-mass-shell. For example, in the transition $N+N \rightarrow N+N^{*}$ one has $\left(q^{\prime}-q\right)^{2}=m_{\mathrm{B}}^{2}$ for $\underset{\sim}{q}=\underset{\sim}{q^{\prime}}$ and

$$
q_{\sim}^{2}=\left[\left(4 m_{\mathrm{B}}{ }^{2}+M_{0}{ }^{2}+M^{* 2}\right)^{2}-4 M_{0}{ }^{2} M^{* 2}\right] / 16 m_{\mathrm{B}}{ }^{2}
$$

if $M^{*}-M \geqq 2 m_{\mathrm{B}}$. Thus we meet again an unphysical complex potential for some exchange and transition interactions. Here we would like to mention that a similar choice for $q_{0}$ is given by Partovi and Lomon ${ }^{17}$

$$
q_{0}=\left(M_{n_{1}}^{2}-M_{n_{2}}^{2}\right) / P_{0}
$$

which leads to the same problems as mentioned before. In view of this pathology which also originates from the different energies of the two particles in unequal mass configurations this approach should be discarded, too.

(iii) Both particles have equal energy, i.e., the relative energy vanishes: $q_{0}=0$. This is achieved by setting

$$
\alpha_{n}=E_{n_{1} q} /\left(E_{n_{1} q}+E_{n_{2} q}\right), \quad \beta_{n}=0 .
$$

It is not surprising that this choice corresponds to the relativistic center-of-mass relative variables for the equal time situation. For configurations $n$ where

1 H. Arenhövel and H. J. Weber, Springer Tracts in Modern Physics, Vol. 65, 58 [1972] ; L. S. Kisslinger, Proc. Topical Meeting on High Energy Collisions, Trieste 1974.

2 H. Arenhövel, Proc. Int. Symposion on Interaction Studies in Nuclei, Mainz, 1975, ed. H. Jochim and B. Ziegler (North-Holland, Amsterdam 1975), p. 727; H. Arenhövel, Proc. I. Int. School of Electro- and Photonuclear Reactions, Erice (1976) (to be published).

${ }^{3}$ H. J. Weber, Proc. Int. Symposion on Interaction Studies in Nuclei, Mainz, 1975, ed. H. Jochim and B. Ziegler, North-Holland, Amsterdam 1975, p. 749 ; H. J. Weber, Proc. VII. Int. Top. Conf. on Meson-Nuclear Physics, P. Barnes and L. S. Kisslinger, eds. (Carnegie-Mellon U., 1976) H. J. Weber and H. Arenhövel (to be published). both particles have the same mass, one has $\alpha_{n}=1 / 2$. The nice feature of this choice is, that the oneboson-exchange part of the potentials, in particular of the transition potentials (e.g. $N+N \rightarrow N^{*}+N$, $N+N \rightarrow N^{*}+N^{*}$ ), is of Yukawa-type with the normal range given by the mass of the exchanged boson. No retardation effects occur and all diagonal and transition potentials are real. In particular, in the nonrelativistic limit the transition potentials which have been used up to now in the nonrelativistic treatment are recovered. Furthermore, the symmetric treatment of the particles distributing the total energy evenly between them is quite appealing.

The foregoing discussion shows that for a relativistic description of isobar configurations in the two-nucleon system starting from a generalized BetheSalpeter equation and approximating it to a threedimensional equation one should give both particles the same energy, i.e., fix the relative energy to zero, irrespective of the fact that they could be in a virtually state. Two other prescriptions for fixing the relative energy lead to serious problems because of the large mass differences of the various isobar configurations involved. A detailed study of this relativistic model for the deuteron and for the nucleon-nucleon scattering below pion threshold is under way and will be reported elsewhere.

\section{Acknowledgement}

Interesting discussions with the late K. Erkelenz, with G. E. Brown, A. D. Jackson and W. Weise are gratefully acknowledged. Furthermore, I thank G. E. Brown for his kind hospitality at SUNY at Stony Brook during August 1975 where part of this investigations has been carried through and the Deutsche Forschungsgemeinschaft for the financial support of this visit.

${ }^{4}$ C. P. Horne et al., Phys. Rev. Lett. 33, 380 [1974]; H. Braun et al., Phys. Rev. Lett. 33, 312 [1974] ; P. Benz and P. Söding, Phys. Lett. 53 B, 367 [1974]; M. T. Emms et al., Phys. Lett. 52 B, 372 [1974].

5 W. Fabian, H. Arenhövel, and H. G. Miller, Z. Phys. 271, 93 [1974]; H. Arenhövel, W. Fabian, and H. G. Miller, Phys. Lett. 52 B, 303 [1974]; W. Fabian and H. Arenhövel, Nucl. Phys. A 258, 461 [1976].

6 A. M. Green and J. A. Niskanen, Nucl. Phys. A 249, 493 [1975]; K. Holinde and R. Machleidt, preprint, Bonn 1975; B. D. Day and F. Coester, Phys. Rev. C 13, 1720 [1976].

7 H. Arenhövel, M. Danos, and H. T. Williams, Nucl. Phys. A 162, 12 [1971] ; S. Jena and L. S. Kisslinger, Ann. Phys. (N. Y.) 85, 251 [1974]; P. Haapakoski and M. Saarela, Phys. Lett. 53 B, 333 [1974]. 
8 H. J. Weber, Phys. Lett. 56 B, 21 [1975], Nucl. Phys. A 264, 365 [1976].

9 H. Arenhövel, Z. Phys. A 275, 189 [1975].

10 A detailed review is given by N. Nakanishi, Progr. Theoret. Phys. Suppl. 43, 1 [1969].

11 M. H. L. Pryce, Proc. Roy. Soc. A 195, 62 [1948].

12 M. Chemtob and M. Rho, Nucl. Phys. A 163, 1 [1971]; M. Chemtob, J. W. Durso, and D. O. Riska, Nucl. Phys. B 38, 141 [1972].
13 R. M. Woloshyn and A. D. Jackson, Nucl. Phys. B 64, 269 [1973].

14 F. Gross, Phys. Rev. 186, 1448 [1969].

15 K. Erkelenz and K. Holinde, Z. Naturforsch. 28 a, 353 [1973].

16 R. Blankenbecler and R. Sugar, Phys. Rev. 142, 1051 [1966].

17 M. H. Partovi and E. L. Lomon, Phys. Rev. D 2, 1999 [1970]. 